\title{
REVIEW
}

\section{The Use of Prospective Meta-Analysis}

\author{
Tarık Yarılgaç ${ }^{1}$ \\ ${ }^{1}$ Ordu University, Faculty of Agriculture, Department of Horticulture, Ordu, Turkey,
}

Received: 14 December 2018, Accepted 20 December 2018, Published online: 27 December 2018

(C) Ordu University Institute of Health Sciences, Turkey, 2018

\begin{abstract}
Meta-analysis is a powerful statistical instrument to summarize the knowledge in a research field, and to estimate overall measures of effects based on reported or unreported results. In performing meta-analysis is generally used the results of the reported trials. Knowing the results before the meta-analysis may affect the description of the review query, the entry and exclusion specifications because the researchers may also be prejudiced in selecting favor of reports supportive for their own thoughts. With these problems the retrospective meta-analysis is becoming a controversial tool in terms of "bias". The prospective metaanalysis can cope with these problems of the retrospective meta-analysis. A prospective meta-analysis is a type of next-generation systematic reviews where studies are investigated to be eligible before reporting their original studies. Prospective Meta-Analysis is unaware of the results of all studies because of the prospective identification and application of selection criteria for trials. In this paper, it is aimed to give information about Prospective Meta-Analysis, and to promote the use.
\end{abstract}

Key words: Meta-Analysis, Prospective Meta-Analysis, Randomized controlled trial, Publication bias, Researcher bias

Address for correspondence/reprints:

Tarık Yarılgaç

Telephone number: +90 (452) 2265200

E-mail: yarilgac@hotmail.com

DOI: $10.19127 / \mathrm{mbsjohs} .497064$

\section{Introduction}

The combining the results of studies on the same subject is continuing for long time as a scientific tradition (Berlin and Colditz, 1999). The utilization of meta-analysis is becoming increasingly prevalent for combine methodological and formally similar studies.

Meta-analysis, a notion coined by Glass (1976), is purposed to ensure the statistical investigation of a wide compilation of analysis outcomes from independent researches for the aim of combination the outcomes. Meta-analysis, or research synthesis, or research combination is a scientific method of achieving this goal through certain statistical methods, and really it has a long and former history (Hartung et al., 2008).

Meta-analysis, which is a complicated statistical method containing the generation of data obtained from related researches to determine impact size or result, has more and more noticed and influence evidence-based medicine, particularly in the area of health sciences. Through the arrival and unfulfilled 
needs of the evidence-based medicine, the initial registerable article of a meta-analysis that addresses the efficiency of the typhoid vaccine in 1904, the quality and number of meta-analyses published in relative to health sciences have increased significantly (Mak et al., 2010).

Upon the emergence of evidence-based medicine approach in the recent two decades, metaanalysis has turned into a widespread study instrument to compound inputs from different studies and process them collectively. Metaanalysis ensures crucial data for creating clinical outlines and generating health policy suggestions (Turok et al., 2011; Tu and Faggion, 2012). Conventional meta-analysis, however, suffers from the heterogeneousness of the including studies of where tests a distinctive interference and evaluates a particular main result. While attempting to include all relevant data, other traps associated with metaanalysis contain discriminative reporting biases and reluctance of investigators' project data (Turok et al., 2011).

Assembling the results of many randomized studies in a meta-analysis ensures a strong and systematic tool to reliably predict modest but valuable developments in therapy. However, traps in the meta-analysis can further be run across because of the strength of this approach in determining small biases. Some of the traps in metaanalysis contain:

- the selection bias of patients;

- the selection bias of studies;

- the bias owing to the post hoc choice of study queries, suitability criteria, result description, or subgroups (Simes, 1995).

\section{Bias in Meta-Analysis}

Meta-Analysis is a statistical method used by researchers to quantify methodologically similar studies. In other words, Meta-analysis is a quantitative systematic review used "to combine the results of a number of different reports into one report to create a single, more precise estimate of an effect" (Ferrer, 1998). With the Meta-Analysis developed as an alternative to traditional literature review, new conclusions are generally made from the results of the previous research. Before applying the meta-analysis, the researchers decide which studies and which statistical methods to use. In this decision phase, they learn the results of previous studies, and this may cause the selection to be made in favor of the own ideas. This potential case arises the problem of bias in meta-analysis (Eysenck, 1994; Egger, 1998).

Typical retrospective meta-analysis looks like investigative rather than corroborative study. Decisions regarding researches to be subsumed, statistical investigations, and moderating elements are generated after the researchers recognize the results of the researches. These retrospective decisions have high probability for bias. For ensure confirmatory proof, methodological decisions in meta-analysis must be carried out prospectively, before the outcomes of the studies are known and ideally before studies are made (Watt and Kennedy, 2017). Multi-center randomized controlled trials and meta-analysis are the gold standard for evidenced-based medicine. However, multi-center trials are expensive and relatively uncommon in the literature. Recently, the use of meta-analysis is popular, because it combines inputs from many trials and processes them in the aggregate. Yet, selective reporting biases when trying to incorporate all thematic input (Turok et al., 2011).

In the meta-analysis, bias is a very important issue. The potential sources of publication bias are "researcher bias", "selection bias of subjects and trials" and "bias due to post hoc selection of study questions", "eligibility criteria", "outcome definitions or subgroups" (Alderson et al., 2004; Askie et al., 2011). Publication bias may cause critical results, particularly in the areas of epidemiology and medicine (Weiss and Wagner, 2011). Dickersin (2005) notes that there are some studies that is influenced by meta-analyses that suffer from publication biases in the even the treatment of life-threatening diseases (Weiss and Wagner, 2011).

\section{Prospective Meta-Analysis}

A meta-analysis may be performed by retrospective or prospective. While, a retrospective meta-analysis is performed on data extracted from the literature, a prospective meta-analysis (PMA) is performed on the actual raw data from the various studies. "Patient-level data" that have been directly and individually collected during a clinical trial as individual will only be available in a prospective analysis (Herson, 2009). This chance of using patient-level data is one of the advantages of PMA.

PMA analysis plans are unaware of the results of all studies. This prevent possibly biased, datadependent emphasis on specific subgroups or specific endpoints. Most widely, PMA has been 
practiced to randomized studies, but it is a method that could be practiced to the PMA of observational studies as well (Berlin and Ghersi, 2004). They have joint properties with both aggregate meta-analyses

and including individual patient data. PMA may help to tackle some of the known troubles of retrospective meta analyses;

- indicating hypotheses that are unaware of the results of individual studies;

- to ensure that the study election criteria are implemented prospectively;

- to ensure that a priori declaration of planned analysis, including subgroup analyses, to be generated before the outcomes of individual studies are known. This prevent probable challenges in explication relevant to the data-dependent emphasis on specific subgroups (Ghersi et al., 2011).

PMA is a meta-analysis where studies (generally randomized controlled trials) are defined, evaluated and specified to be suitable before the results of any of the studies become known. This is distinct from a systematic review because the included studies are usually determined after completion and reporting of results (Ghersi et al., 2011; Anonymous 2, 2018; Anonymous 2, 2018)

Retrospective meta-analysis rises the statistical strength and ensure more exact predictions of the therapy influence by incrementing sample size. While meta-analysis use widespread in medical literature, PMA is a comparatively new methodology. In PMA, the certain interferences as well as the primary and secondary results are stated before data from certain studies are published (Turok et al., 2011). PMAs enable the identification of hypotheses before the results of individual studies, and provide a prospective execution of study selection criteria; and perform the predefinitions of the intended analyses. PMAs are generally undertaken by a common group and generally collect and analysis individual patient data (Ghersi et al., 2011).

Watt and Kennedy (2017) reported that there are three options for PMA:

1. The most apparent choice is to pre-register the meta-analysis plan and include in the meta-analysis only researches handled after the plan was registered. The meta-analysis plan would determine the statistical investigations and the criteria for detecting which studies are included. Next studies that abide by the inclusion/exclusion criteria would be included in the meta-analysis. Unhappily, that choice is probably to keep remarkable retrospective deciding.

2. A more powerful alternative for PMA is to pre-determine the protocols of studies included as part of the meta-analysis plan. In multicenter studies, all researchers generally need to adopt the same protocols.

3. It is recommended that a registration-based PMA in which the decision to include or exclude a specific research study prospectively based on preliminary records for the research.

PMA ensures statistical strength to study substantial queries about rare cases and subgroups. The skill to examine subgroups is not specific to PMA, but a pre-description of subgroups in a PMA submits the benefit of preventing the bias in the post hoc description of prevalently used subgroups (Rothstein et al., 2005).

A PMA must be planned meticulously and should be registered a protocol such as that sustained by the Cochrane Collaboration from the outset (Reade et al, 2010; Anonymous 8, 2018). There are several PMAs maintained over decades, with new trials adding to the enlarging data compile (Porgue and Yusuf, 1998). If the meta-analysis is finished prematurely, there is little instance to conduct the reactions of researchers in trials still underway (Reade et al., 2010).

A PMA should have a openly obtainable guideline. Berlin and Ghersi, (2004) and Ghersi et al. (2011) note that the contents of this protocol can be summarized as in Table 1 .

\section{An Example for the Use of PMA}

Askie et al. (2018) performed a study to compare the influences of varying target intervals for oxygen saturation as determined via pulse oximetry ( $\mathrm{SpO} 2)$ on major morbidity or death. A prospectively planned meta-analysis named as Neonatal Oxygenation Prospective Meta-analysis (NeOProM) composed of independent participators' input for five clinical trials (Anonymous 1-6, 2018) These researches were assessed as suitable to include in the meta-analysis before the outcomes of any of the trials given (Ghersi et al. 2011). 4965 newborns were included to this study. The study protocol was published in 2011 and registered on "ClinicalTrials.gov". The statistical analysis plan was finalized in 2015. In this PMA results of independent participators' input from highly preterm newborns, no significant difference was detected in a comparison between 


\section{REVIEW}

lower $\mathrm{SpO} 2$ target interval and higher $\mathrm{SpO} 2$ target interval on the early combined result of major disability or death at an adjusted age of 18 to 24 months. The lower $\mathrm{SpO} 2$ target interval was related with an elevated threat of necrotizing enterocolitis and death, however a lower risk of retinopathy during premature therapy. All in all, the use of PMA provided in this study that the researchers can comply to take advantage of the identical tool to define a specific result, and to determine the results at the identical check-points in the trials.

Table 1. Contents of a PMA as stepwise (Berlin and Ghersi, 2004)

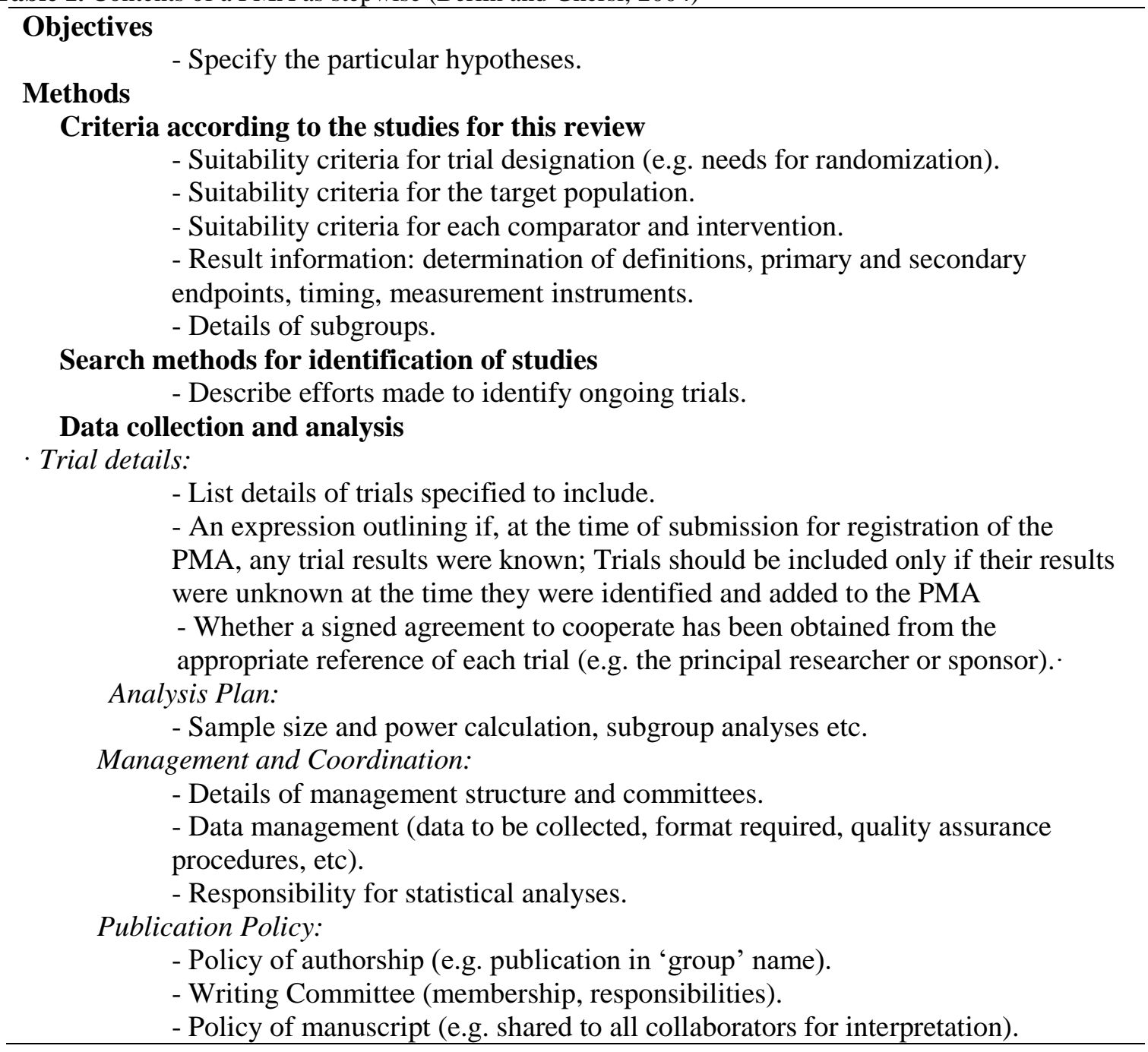

\section{Conclusion}

In this paper, it is aimed to give information about PMA, a type of next-generation systematic reviews, and to promote the use.

In recent years, the use of meta-analysis has become increasingly popular, as it has ability to combining the results of different studies in one study. However, publication bias is a serious problem in meta-analysis, which can affect statistical power. Thus, PMA with some distinct advantages should be preferred rather than retrospective meta-analysis of published data.
As a result, PMA can used in the medical research to establish causative links between treatment and results. Planning a PMA of collected data from associated researches and the usage of real data will help a more accurately prediction of the influences of treatment methods in health sciences. 
Peer-review: Externally peer-reviewed.

Author Contributions: Concept- T. Y., Design- T. Y., Supervision- T. Y., Literature Review- T. Y., Writing- T. Y., Critical Review- T. Y.

Conflict of Interest: No conflict of interest was declared by the author.

Financial Disclosure: The author declared that this study hasn't received no financial support.

\section{References}

Alderson P, Green S, Higgins JPT, Eds. The Cochrane Library, Issue 1. Chichester, UK: John Wiley \& Sons, Ltd; 2004. Cochrane Reviewers' Handbook 4.2.2 [Updated March 2004].

Anonymous 1. Clinical Trials website. Surfactant Positive Airway Pressure and Pulse Oximetry Trial (SUPPORT). Available from: https://clinicaltrials.gov/ct2/show/NCT0023332 4. Accessed 15.09.2018.

Anonymous 2. Cochrane Methods. 2018. Assessing risk of bias in included studies. Available from: http://methodscochraneorg/bias/assessing-riskbias-included-studies. Accessed 27.05.2018).

Anonymous 3. Current Controlled Trials website. Efficacy and safety of targeting lower arterial oxygen saturations to reduce oxygen toxicity and oxidative stress in very preterm infants: The Canadian Oxygen Trial. Available from:http://www.isrctn.com/ISRCTN62491227. Accessed 15.09.2018.

Anonymous 4. Current Controlled Trials website. Which oxygen saturation level should we use for very premature infants? a randomised controlled trial. Available from: http://www.isrctn.com/ISRCTN00842661.

Accessed 15.09.2018.

Anonymous 5. Australian New Zealand Clinical Trials Registry website. A randomised phase III study to evaluate whether a lower versus a higher oxygen saturation target in infants of $<28$ weeks gestation is associated with a reduction in death or disability at 2 years of age. Available from:http://www.anzctr.org.au/ACTRN126050 00253606.aspx. Accessed 15.09.2018.
Anonymous 6. Australian New Zealand Clinical Trials Registry website. Which oxygen saturation level should we use for very premature infants? a randomized controlled trial to investigate the effect of two slightly different oxygen levels on the health of very premature infants.

Available from:http://www.anzctr.org.au/ACTRN126050 00055606.aspx. Accessed 15.09.2018.

Anonymous 7. Prospective meta analysis. Nottingham Clinical Trials Unit. Available from: https://www.nottingham.ac.uk/nctu/otherresearch/pretermbirth/projects/prospectivemeta-analysis.aspx. (Accessed 19.08.2018).

Anonymous 8 . How to plan and execute a PMA. Available from: https://methods.cochrane.org/pma/how-planand-execute-pma. (Accessed 10.12.2018).

Askie LM, Brocklehurst P, Darlow BA, Finer N, Schmidt B, Tarnow-Mordi W, NeOProM Collaborative Group. NeOProM: neonatal oxygenation prospective meta-analysis collaboration study protocol. BMC Pediatr. 2011; 11:6.

Askie, L.M., Darlow, B.A., Finer, N., Schmidt, B., Stenson, B., Tarnow-Mordi, W. et al. Association between oxygen saturation targeting and death or disability in extremely preterm infants in the Neonatal Oxygenation Prospective Meta-Analysis Collaboration. (for the; Neonatal Oxygenation Prospective Meta-analysis (NeOProM) Collaboration) JAMA. 2018; 319:2190-2201.

Berlin JA, Colditz GA. The role of meta analysis in the regulatory process for foods, drugs and devices. Journal of the American Medical Association 1999;281(9):830-834.

Berlin JA, Ghersi D. Prospective meta-analysis in dentistry. J. Evid. Base Dent. Pract. 2004; 4:5964.

Dickersin K. Publication bias: Recognizing the problem, understandings its origins and scope, and preventing harm. In H.R. Rothstein, A. J. Sutton, \& M. Borenstein (Eds.), Publication bias in meta-analysis: Prevention, assessment, and adjustments, West Sussex: Wiley 2005;11-34.

Egger M, Davey-Smith G. Bias in location and selection of studies. BMJ 1998; 316:61-6

Eysenck HJ. Meta-analysis and its problems. BMJ 1994; 309:789-92.

Ferrer RL. Graphical methods for detecting bias in meta-analysis. Family Med 1998; 30:579-83. 


\section{REVIEW}

Ghersi D, Berlin J and Askie L. "Chapter 19: Prospective meta-analysis," In Cochrane Handbook for Systematic Reviews of Interventions Version 5.1.0 2011; Available from: https://handbook-5-1.cochrane.org (Accessed: 10.08.2018)

Glass G. Primary, secondary, and meta-analysis of research. EducRes 1976; 5:3-9.

Hartung J, Knapp G and Sinha BK. Statistical metaanalysis with applications. John Wiley \& Sons, Inc. 2008, Hoboken, New Jersey.

Herson J. Data and safety monitoring committees in clinical trials. Boca Raton, FL: Chapman \& Hall/CRC; 2009.

Mak A, Cheung MWL, Fu EHY, Ho RCM. Metaanalysis in medicine: an introduction. International Journal of Rheumatic Diseases 2010; 13:101-104.

Pogue J, Yusuf S. Overcoming the limitations of current meta-analysis of randomized controlled trials. Lancet. 1998;3(351):47-52.

Reade MC, Delaney A, Bailey MJ, et al. Prospective meta-analysis using individual patient data in intensive care medicine. Intensive Care Med. 2010; 36:11-21.

Rothstein HR, Sutton AJ, Borenstein M. Publication bias in meta-analysis: prevention, assessment and adjustments. John Wiley \& Sons Ltd. 2005 research. ISRN Dent. 2012; 2012:10.

Simes RJ. Prospective meta-analysis of cholesterollowering studies: The Prospective Pravastatin Pooling (PPP) Project and the Cholesterol Treatment Trialists (CTT) Collaboration. American Journal of Cardiology 1995;76(9):122-126.

$\mathrm{Tu}$ Y-K, Faggion CM. A primer on network metaanalysis for dental. ISRN Dentistry, 2012:1-10. doi:10.5402/2012/276520

Turok DK, Espey E, Edelman AB, Lotke PS, Lathrop EH, Teal SB, Jacobson JC, Simonsen SE, Schulz KF. The methodology for developing a prospective meta-analysis in the family planning community. Trials $2011 ; 12: 104$.

Watt CA, Kennedy JE. Options for prospective meta-analysis and introduction of registrationbased prospective meta-analysis. Frontiers in Psychology 2017;7(2030).

Weiss B, Wagner M. The Identification and Prevention of Publication Bias in the Social Sciences and Economics. Journal of Economics and Statistics (Jahrbuecher fuer Nationaloekonomie und Statistik), De Gruyter. 2011;231(5-6):661-684. 